\title{
ESTUDO DA VARIAÇÃO NEUTROPÊNICA PELO SULFATO DE VINCRISTINA EM CÃES COM TVT TRATADOS EM UM HOSPITAL VETERINÁRIO DO NOROESTE PAULISTA
}

\author{
Adriana Antonia Cruz Furini ${ }^{1}$ \\ Paula Mateus Guimarães \\ Fernanda Santos Silva ${ }^{1}$ \\ Marcela Aparecida Sousa Moreno Lopes ${ }^{1}$ \\ Karina Ferreira Castro ${ }^{1}$ \\ Tábata Salum Calile Atique \\ Halim Atique Netto ${ }^{1}$
}

FURINI, A. A. C.; GUIMARÃES, P. M.; SILVA, F. S.; LOPES, M. A. S. M.; CASTRO, K. F. ATIQUE, T. S. C.; ATIQUE NETTO, H. Estudo da variação neutropênica pelo sulfato de vincristina em cães com TVT tratados no hospital veterinário do noroeste paulista. Arq. Ciênc. Vet.Zool. UNIPAR, Umuarama, v. 17, n. 1, p. 5-9, jan./mar. 2014.

RESUMO: O presente estudo avaliou dados epidemiológicos de neutropenia induzida por sulfato vincristina em cães com Tumor Venéreo Transmissível (TVT), num Hospital Veterinário do Noroeste paulista. Para tanto, trata-se de um estudo do tipo retrospectivo de protocolos eletrônicos e formulários manuais de dados digitalizados de 51 casos de TVT. O atendimento ambulatorial e de internação desses animais foi realizado no período de setembro de 2006 a dezembro de 2009 . Dentre os resultados, destaca-se que 51 animais foram diagnosticados com TVT, sendo 37 fêmeas (73\%) e 14 (27\%) machos; 46 animais $(90,2 \%)$ foram tratados exclusivamente com sulfato de vincristina. Os cães Sem Raça Definida-SRD (n=28) foram os maiores acometidos com 54,9\%; seguidos pelos Poodles com quatro cães (7,84\%). Os animais com idade entre 37 e 84 meses obtiveram a maior porcentagem de acometimento pelo TVT com 20 casos $(39,22 \%)$. Em doze animais $(23,52 \%)$ foi observada neutropenia (valores entre 390 a 1927 cél/ $\mu \mathrm{L}$ ). Conclusão: a possível toxicidade medular induzida pela vincristina foi verificada pela descrição da neutropenia. Dessa forma, a identificação de quadros neutropênicos, por meio da realização de hemogramas semanais, é considerada obrigatória e de extrema relevância devido à prevalência de mielotoxicidade secundária à utilização deste quimioterápico.

PALAVRAS-CHAVE: Cães. Neutropenia. Sulfato de Vincristina. TVT.

\section{NEUTROPENIC VARIATION BY VINCRISTINE SULFATE IN TVT DOGS TREATED IN A VETERINARY HOSPITAL IN NORTHWESTERN SÃO PAULO}

\begin{abstract}
Objective: The present study aims to assess epidemiological data on neutropenia induced by vincristine sulfate among dogs with Canine Transmissible Venereal Tumor (CTVT) at a veterinary hospital in the Northwestern region in the São Paulo State. Methodology: This is a retrospective study of electronic protocols and digitalized data from manually filled form from 51 CTVT cases. These animals were treated in an outpatient care clinic and hospitalization took place from September 2006 to December 2009. Results: 51 animals were diagnosed with CTVT; among these, 37 were female (73\%) and 14 were male (27\%). From these, 46 animals $(90.2 \%)$ were treated exclusively with vincristine sulfate. Among them, mongrels $(n=28)$ were the most common, with 54.9\%, followed by Poodles, with 4 dogs $(7.84 \%)$. Animals aged from 37 and 84 months were the largest age group, with 20 cases (39.22\%). Neutropenia (390 to 1927 cells/ $\mu \mathrm{L}$ ) was observed in 12 animals. Conclusion: possible vincristine-induced marrow toxicity was verified by the description of neutropenia. Thus, neutropenia identification through weekly blood count cells is considered extremely important and mandatory due to the prevalence of myelotoxicity following treatment with this chemotherapeutic drug.
\end{abstract}

KEYWORDS: Dogs. Neutropenia. Vincristine Sulfate. TVT

\section{ESTUDIO DE LA VARIACIÓN NEUTROPÉNICA POR SULFATO DE VINCRISTINA EN PERROS CON TVT TRATADOS EN EL HOSPITAL VETERINARIO DEL NOROESTE PAULISTA}

RESUMEN: Este estudio busca evaluar datos epidemiológicos de neutropenia inducida por sulfato vincristina en perros con Tumor Venéreo Transmisible (TVT), en un Hospital Veterinario del Noroeste Paulista. Es un estudio del tipo retrospectivo de protocolos electrónicos y formularios manuales de datos digitalizados de 51 casos de TVT. El atendimiento de ambulatorio y de internación de esos animales se realizó en el período de septiembre de 2006 a diciembre de 2009. Entre los resultados, se destaca que 51 animales fueron diagnosticados con TVT, siendo 37 hembras (73\%) y 14 (27\%) machos; 46 animales $(90,2 \%)$ fueron tratados exclusivamente con sulfato de vincristina. Los perros Sin Raza Definida - SRD ( $\mathrm{n}=28$ ) fueron los más acometidos con 54,9\%; seguidos por los Poodles con cuatro perros (7,84\%). Los animales con edad entre 37 y 84 meses obtuvieron mayor porcentaje de acometimiento por TVT, con 20 casos $(39,22 \%)$. En doce animales $(23,52 \% 0$ se ha observado neutropenia (valores entre 390 a 1927 cél/ $\mu \mathrm{L}$ ). Conclusión: la posible toxicidad medular inducida por vincristina se ha

DOI: https://doi.org/10.25110/arqvet.v17i1.2014.4911

${ }^{1}$ Centro Universitário de Rio Preto - UNIRP. Rua Yvette Gabriel Atique, 45, Boa Vista, São José do Rio Preto - SP. E-mail: adriana.cruz.furini@gmail.com 
verificado por la descripción de la neutropenia. Así, la identificación de cuadros neutropénicos por medio de realización de hemogramas semanales, es considerada obligatoria y de extrema relevancia debido a la prevalencia de mielotoxicidad secundaria a la utilización de este quimioterápico.

PALABRAS CLAVE: Perros. Neutropenia. Sulfato de Vincristina. TVT.

\section{Introdução}

A prevalência de neoplasias em cães tem aumentado de maneira considerável nos últimos anos, e estima-se que um em quatro cães com idade superior a dois anos, morra de câncer (MORRIS; DOBSON, 2007; PAOLONI; KHANNA, 2007). O TVT é uma neoplasia de células redondas, que mais acomete a espécie canina (SILVA et al., 2007). Essa neoplasia ocorre com maior frequência em cães jovens errantes e sexualmente maduros com idade entre 4 e 5 anos; e em diferentes raças, fazendo parte do grupo de risco os cães sem raça definida (SRD) e os cães abandonados (DAS; DAS, 2000; NAK et al., 2005; CAVALCANTI et al., 2007, WITHROW; MACEWEN, 2007). Além disso, apresenta distribuição mundial, sendo mais comum em regiões tropicais e subtropicais (SILVA et al., 2007; WITHROW; MACEWEN, 2007).

Apresenta-se na mucosa genital externa de cães de ambos os sexos, entretanto tem sido descrito em várias localizações extragenitais (WITHROW; MACEWEN, 2007; SILVA et al., 2007). Quanto a sua localização, no macho, situa-se no parte caudal do pênis, na área da glande e, ocasionalmente, no prepúcio. Na fêmea é normalmente encontrada na parte posterior da vagina e muitas vezes, na junção do vestíbulo. Aproximadamente $5 \%$ dos casos evoluem para metástase, com disseminação para a pele, linfonodos regionais, tonsílas, olhos, cérebro, hipófise, plano nasal, língua e lábios (DAS; DAS, 2000; NAK et al., 2005). Embora diversas modalidades de tratamento sejam propostas, a quimioterapia com sulfato de vincristina é considerada eficaz e menos tóxica do que outras intervenções (NAK et al., 2005; RODASKI; DE NARDI, 2007); e amplamente aceita na Medicina Veterinária para o tratamento de cães (RODASKI; DE NARDI, 2007; CHUN, 2009).

Diversas modalidades de tratamento são propostas, tais como a excisão cirúrgica, radioterapia e a imunoterapia, entretanto a quimioterapia com sulfato de vincristina é considerada eficaz, menos tóxica e amplamente aceita na medicina veterinária (NAK et al., 2005; RODASKI; DE NARDI, 2007; CHUN, 2009).

A vincristina (Vinca rosea Linn), embora considerada um fármaco relativamente bem tolerado e eficaz nos cães, tem apresentado efeitos citostáticos indesejáveis e não seletivos, principalmente nas células sanguíneas e nas células hematopoiéticos, causando mielossupressão (NAK et al., 2005; RODASKI; DE NARDI, 2007; FARO et al., 2008; CHUN, 2009). As células com menor tempo de vida circulante são as mais susceptíveis, como os neutrófilos e as plaquetas (WITHROW; MACEWEN, 2007).

O nadir neutrófilico, definido como ponto em que o fármaco atinge o seu efeito máximo nas linhagens sanguíneas geralmente ocorre após sete dias de uma sessão de quimioterapia. Já a neutropenia geralmente persiste por três a cinco dias, e em pouco menos de duas semanas, a celularidade da médula óssea passa por uma fase de recuperação (LANORE; DELPRAT, 2004). Devido a ocorrência de leucopenia secundária à utilização dos quimioterápicos faz-se necessário o monitoramento laboratorial semanal dos animais que utilizam esse tipo de medicamentos (FARO et al., 2008).

No Hospital Veterinário "Dr. Halim Atique", interior paulista, a medicação quimioterápica é adquirida em clínica especializada para manipulação de medicamentos citotóxicos (quimioterápicos), sendo o serviço terceirizado, conforme a Resolução RDC 220 de 21 de setembro de 2004 da Agência Nacional de Vigilância Sanitária (ANVISA). Essa padronização do serviço foi o principal incentivo para realização do presente estudo com avaliação de dados epidemiológicos de TVT e secundariamente de casos neutropenia induzida por vincristina em cães.

\section{Material e Métodos}

O estudo realizado foi do tipo retrospectivo, por meio de protocolos eletrônicos e formulários manuais. Os dados foram digitalizados em tabela no formato Excel, referentes ao período de setembro de 2006 a dezembro de 2009. Esses animais eram cães atendidos no ambulatório do Hospital Veterinário 'Dr. Halim Atique', Centro Universitário de Rio Preto, São Paulo, Brasil. Foram excluídos do estudo cães com outro tipo de neoplasia associada ou não ao TVT.

Os critérios de inclusão foram as amostras de TVT de pacientes canino de ambos os sexos, sem distinção de raça e idade, com sinais clínicos compatíveis com TVT, encaminhadas pelo corpo clínico ao laboratório de anatomia patológica, para realização dos exames citológicos.

Paralelamente, foram analisados nos prontuários o protocolo quimioterápico utilizado e as variações dos neutrófilos a cada semana, durante todo o tratamento. Valores dos neutrófilos abaixo de 1.500 células/ $\mu \mathrm{L}$ foram utilizados como valores de corte das taxas de neutrófilos para decisão pela quimioterapia.

O volume de sangue utilizado para a contagem total dos leucócitos foi de $12 \mu \mathrm{L}$, sendo a contagem realizada no aparelho Horiba Medical (modelo ABX Micros ABC VE), enquanto, a contagem diferencial foi realizada manualmente. Os exames foram realizados dois dias antes do tratamento e cinco dias após a administração do quimioterápico. O nadir neutrofílico foi considerado cinco dias após a quimioterapia (LANORE; DELPRAT, 2004).

O programa BioEstat versão 5.0 (AYRES et al., 2008) foi utilizado para análise estatística, mediante a utilização do teste Qui-quadrado e teste Exato de Fisher. O valor de $\mathrm{p}$ menor que $0,05(\mathrm{p}<0,05)$ foram considerados estatisticamente significantes.

\section{Resultados}

Setenta e dois cães realizaram tratamento utilizando protocolos de quimioterapia, no período de janeiro de 2006 a dezembro de 2009. A neoplasia mais frequente foi o TVT da genitália externa, com 70,83\% ( $\mathrm{n}=51)$, seguido do linfoma $12,5 \%(n=9)$, e mastocitoma 8,33\% $(n=6)$.

Em relação aos animais com TVT, 37 (73\%) eram 
fêmeas e 14 (27\%) machos, com proporção de fêmea:macho de 2,64; porém sem significância estatística $(p=0,4573)$. Em relação à raça, os cães SRD foram os mais acometidos $(54,90 \%)$, seguidos pelos Poodles com 7,84\% $(n=3)$ e pelos Labradores com 5,88\% ( $n=3)$. Os resultados não foram estatisticamente significativos $(\mathrm{p}>0,6390)$ para a distribuição dos cães, quando avaliado raça versus sexo (Tabela 1).

Tabela 1: Distribuição de casos de cães com TVT $(n=51)$ de acordo com sexo e raça, submetidos ao tratamento com sulfato de vincristina no Hospital Veterinário Dr. Halim Atique, no período de setembro de 2006 a dezembro de 2009.

\begin{tabular}{|c|c|c|c|}
\hline \multirow[t]{2}{*}{ Raça } & \multicolumn{2}{|c|}{ Sexo } & $\begin{array}{c}\text { Frequência } \\
\text { Absoluta }\end{array}$ \\
\hline & Fêmeas & Machos & $\%$ \\
\hline
\end{tabular}

\begin{tabular}{lccc}
\hline SRD & 20 & 8 & 54,90 \\
BOXER & 2 & - & 3,91 \\
POODLE & 3 & 1 & 7,84 \\
LABRADOR & 3 & - & 5,88 \\
COCKER & 1 & 1 & 3,92 \\
OUTROS & 8 & 4 & 23,53 \\
TOTAL & 37 & 14 & 100 \\
\hline
\end{tabular}

Sem significância estatística

Quanto à faixa etária, foi observado maior índice: 20 $(39,22 \%)$ dos cães que se encontravam entre 37 e 84 meses de idade; $15(29,41 \%)$ acima de 84 meses; 13 (25,49\%) entre 13 e 36 meses de idade. A faixa etária de 37 a 84 (20/39,22\%) meses foi estatisticamente significante $(p=0,0394)$ para a raça Poodle (Tabela 2).

Tabela 2: Distribuição de casos de cães com TVT ( $n=51)$, de acordo com a idade e raça, submetidos ao tratamento com sulfato de vincristina no Hospital Veterinário Dr. Halim Atique no período de setembro de 2006 a dezembro de 2009

\begin{tabular}{|c|c|c|c|c|c|c|c|c|c|c|}
\hline \multirow{3}{*}{ Raça } & \multicolumn{8}{|c|}{ Faixa etária em meses } & \multirow{2}{*}{\multicolumn{2}{|c|}{ Total }} \\
\hline & \multicolumn{2}{|c|}{$0-12$} & \multicolumn{2}{|c|}{$13-36$} & \multicolumn{2}{|c|}{$37-84$} & \multicolumn{2}{|c|}{ Acima de 84} & & \\
\hline & $\mathrm{N}$ & $\%$ & $\mathrm{~N}$ & $\%$ & $\mathrm{~N}$ & $\%$ & $\mathrm{~N}$ & $\%$ & $\mathrm{~N}$ & $\%$ \\
\hline SRD & 3 & 5,88 & 7 & 13,73 & 9 & 17,65 & 9 & 17,65 & 29 & 54,90 \\
\hline Boxer & - & - & 1 & 1,96 & 1 & 1,96 & - & - & 2 & 3,92 \\
\hline Poodle & - & - & - & - & $4 *$ & 7,84 & - & - & 4 & 7,84 \\
\hline Labrador & - & - & 2 & 3,92 & 1 & 1,96 & - & - & 3 & 5,88 \\
\hline Cocker & - & - & 1 & 1,96 & 1 & 1,96 & - & - & 2 & 3,92 \\
\hline Outros & - & - & 2 & 3,92 & 4 & 7,84 & 6 & 11,76 & 12 & 23,53 \\
\hline Total & 3 & 5,88 & 13 & 25,49 & 20 & 39,22 & 15 & 29,41 & 51 & 100 \\
\hline
\end{tabular}

*Exato de Fisher $(\mathrm{p}=0,0394)$

Quarenta e seis animais $(90,20 \%)$ utilizaram o sulfato de vincristina, enquanto, nos demais associou-se o mesmo a ciclofosfamida $(1 / 1,96 \%)$, a asparaginase e ciclofosfamida $(1 / 1,96 \%)$ e doxorrubicina $(3 / 5,88)$.

Não foram observadas alterações na contagem ab- soluta de neutrófilos segmentados entre todas as semanas de quimioterapia, em $39(76,47 \%)$ cães que utilizaram sulfato de vincristina. Doze animais (12/51), 23,52\% apresentaram neutropenia (abaixo de 1.500 cél $/ \mu \mathrm{L}$ - Tabela 3 ).

Tabela 3: Distribuição de cães com diminuição neutrofilica $(n=12)$, em intervalos de cinco dias entre as sessões de quimioterapia com sulfato de vincristina, no período de setembro de 2006 a dezembro de 2009, no Hospital Veterinário Dr. Halim Atique.

\begin{tabular}{|c|c|c|c|c|c|c|c|c|c|c|}
\hline \multicolumn{11}{|c|}{ Neutrófilos - Semanas } \\
\hline Animais & $1^{\mathrm{a}}$ & $2^{\mathrm{a}}$ & $3^{\mathrm{a}}$ & $4^{a}$ & $5^{\mathrm{a}}$ & $6^{\mathrm{a}}$ & $7^{\mathrm{a}}$ & $8^{a}$ & $9^{a}$ & $10^{\mathrm{a}}$ \\
\hline 4 & - & - & - & - & - & 1649 & - & - & - & - \\
\hline 5 & - & $1064 *$ & - & $264^{*}$ & - & $1344^{*}$ & - & - & - & - \\
\hline 15 & - & - & - & $* *$ & - & 1088 & $1292 *$ & $* *$ & - & - \\
\hline 18 & - & - & - & - & 620 & - & - & - & - & - \\
\hline 25 & - & 1701 & - & - & - & - & - & - & - & - \\
\hline 44 & - & - & 1758 & - & - & - & - & - & - & - \\
\hline 49 & - & - & - & - & 1428 & - & - & - & - & - \\
\hline 60 & - & - & - & 1734 & - & - & - & - & - & - \\
\hline 62 & - & - & $630^{*}$ & - & $* *$ & - & - & - & $*$ & - \\
\hline 65 & - & 1890 & - & 1350 & 962 & - & - & - & - & - \\
\hline 68 & - & 1927 & - & - & - & - & - & - & - & - \\
\hline 69 & - & $* *$ & - & 1258 & - & - & 760 & 1435 & - & 390 \\
\hline
\end{tabular}


Dos animais que apresentaram neutropenia entre a primeira e a segunda sessão esse índice foi de 33,33\% $(n=4)$, entre a segunda e terceira sessão foi de $16,67 \%(n=2)$, assim como entre a sexta e sétima sessão. No intervalo entre a terceira e quarta sessão a porcentagem foi de 33,33\% (n=4), e entre a quarta e quinta; e também para quinta e sexta os índices foram iguais a $25 \%(n=3)$.

Do total de 51 animais com TVT, $34(64,71 \%)$ desistiram do tratamento por motivos financeiros, 4 (7,84\%) animais evoluíram para cura com exames citológicos negativos para o TVT. Para nove animais $(17,75 \%)$ não foi possível obter informações dos resultados dos exames citológicos e avaliação de cura. Dois $(3,91 \%)$ animais tiveram alta hospitalar sem a realização do exame citológico. Finalizando, dois $(3,91 \%)$ vieram a óbito durante o tratamento por causas não especificadas no prontuário.

\section{Discussão}

Conforme estudos realizados no Brasil, particularmente em Goiânia, Porto Alegre, e Rio Grande do Norte, as fêmeas são consideradas mais susceptíveis ao TVT (CAVALCANTI et al., 2007; SILVA et al., 2007; SIMERMANN, 2009) devido ao comportamento durante o cio, no qual de maneira geral, ocorre a aceitação de diferentes machos, e também devido à ação hormonal que favorece a implantação das células tumorais (SOBRAL; TINUCCI; CAMACHO, 1998; SILVA et al., 2007). No presente estudo, o maior índice do TVT foi nas fêmeas (73\%), sendo coerente com o referido pela literatura (CAVALCANTI et al., 2007; SILVA et al., 2007; SIMERMANN, 2009). Por outro lado, não foi significativo estatisticamente em relação aos machos, podendo ter sido decorrente do pequeno número de amostras envolvidas.

O maior acometimento do TVT em cães SRD pode estar associado a esses animais pertencerem a famílias de baixas condições socioeconômicas (dado não avaliado) que podem estar criando seus animais em condições de maior promiscuidade (LIMA et al., 2011; SILVA et al., 2007). Embora, na maioria dos estudos, não são encontrados dados significativos para susceptibilidade de determinada raça, mas a incidência esta aumentada nos animais errantes e durante o período de atividade sexual (CRUZ et al., 2009; SILVA et al., 2007, SIMERMANN, 2009).

Devido ao TVT se disseminar com maior incidência em áreas com maior número de cães $\mathrm{SRD}$, faz-se necessário o desenvolvimento de programas preventivos e educativos com objetivo de orientação da população ao controle populacional e sanitário dos cães. Esses podem incluir programas de castração em massa; orientação aos proprietários em relação à forma de vida do cão; identificação e tratamento de animais portadores; e projetos que visem à esterilização e adoção de cães de rua.

Os resultados em relação à faixa etária foram significativos para os animais com idade entre os 37-84 meses com 39,22\% ( $p=0,0394)$, coerente com outros autores que descreveram que o TVT acomete principalmente animais com idade entre quatro e cinco anos de idade (CAVALCANTI et al., 2007), pois essa fase corresponde a maior maturidade e atividade sexual (NAK et al., 2005; CAVALCANTI et al., 2007; SILVA et al., 2007; WITHROW; MACEWEN, 2007; SIMERNANN, 2009; LIMA et al., 2011).
A quimioterapia citotóxica é um método eficiente com baixo número de recidivas e de custo inferior a outros quimioterápicos (LIMA et al., 2007). O sulfato de vincristina na dose de $0,025 \mathrm{mg} / \mathrm{Kg}$ a cada 7 dias por via endovenosa em geral determina a regressão do tecido tumoral após a segunda semana de administração do medicamento, com totalidade na quarta semana (NAK et al., 2005; LIMA et al., 2011). O uso do sulfato de vincristina no tratamento de 46 dos 51 animais com TVT do presente estudo corrobora a unanimidade das referências médico veterinárias sobre a eficácia desse medicamento no tratamento da neoplasia (NAK et al., 2005; SILVA et al., 2007; CAVALCANTI et al., 2007; SIMERNAMM, 2009).

Contudo, a maioria dos agentes quimioterápicos, dentre eles o sulfato de vincristina, possui como alvo as células de intensa divisão celular, agindo em tecidos neoplásicos assim como em tecidos normais, e como consequência, apresenta efeitos adversos. A neutropenia associada aos alcaloides da vinca caracteriza-se como um importante exemplo desses efeitos, podendo acarretar a suspensão do tratamento, infecções graves e óbito (BRANDÃO et al., 2002; RODASKI; NARDI, 2007;).

A avaliação semanal dos hemogramas, seguindo os princípios de O'Keefe e Harris (1990), pode prevenir complicações que justifiquem a interrupção da quimioterapia com o sulfato de vincristina (RODASKI; NARDI, 2007; FARO et al., 2008).

Estudos realizados por Faro et al. (2008) e Anai (2011), em 8 e 25 cães, respectivamente, obtiveram neutropenia da linhagem segmentada. No estudo realizado por Faro et al. (2008), os resultados foram comparadas com outros protocolos no tratamento do TVT: COP1 - vincristina $(0,75$ $\left.\mathrm{mg} / \mathrm{m}^{2}\right)$, ciclofosfamida $\left(300 \mathrm{mg} / \mathrm{m}^{2}\right)$ e prednisona $(1 \mathrm{mg} / \mathrm{kg})$; COP2 - vincristina $\left(0,5 \mathrm{mg} / \mathrm{m}^{2}\right)$, ciclofosfamida $\left(50 \mathrm{mg} / \mathrm{m}^{2}\right)$ e prednisona $(10 \mathrm{mg} / \mathrm{kg})$, ocorrendo significância para diminuição dos neutrófilos segmentados na primeira semana após utilização do protocolo COP1. Por outro lado, Anai (2011) utilizando o sulfato de vincristina no tratamento do linfoma, obtve a presença de neutropenia após a primeira $(31,57 \%)$, terceira $(44,4 \%)$, sexta $(40 \%)$ e oitava sessão de tratamento. No presente estudo, os valores da neutropenia $(26,08 \%)$, utilizando valores menores que $1.500 \mathrm{cél} / \mu \mathrm{L}$, foram maiores que os $8 \%$ citado por Nak et al., (2005), na Túrquia. Contudo, quanto à incidência, os valores foram similares aos da literatura (FARO et al., 2008), citando maior taxa de neutropenia entre a primeira e a segunda sessão.

A neutropenia deve-se ação citostática do sulfato de vincristina, por causar aplasia-hipoplasia medular, sobre o compartimento de diferenciação das células percussoras envolvidas na linhagem dos neutrófilos; além de outras linhagens (PEREZ et al., 2005). A avaliação da toxicidade à medula óssea, por meio da realização de hemogramas semanais, durante o tratamento quimioterápico de animais portadores do TVT, é considerada obrigatória e de extrema relevância. Ademais, devido a sequência sanguínea de declínio na contagem de células estarem relacionadas ao tempo de meia vida dessas, a neutropenia constitui-se na primeira alteração observada. 


\section{Conclusões}

O estudo permite concluir que o TVT apresenta uma prevalência maior em cães SRD $(54,90 \%)$, fêmeas $(73 \%)$ e com idade entre 37 e 84 meses $(39,22 \%)$. O sulfato de vincristina foi o quimioterápico de escolha pelos clínicos veterinários. A toxicidade medular pelo sulfato de vincristina foi verificada pela neutropenia, em $12(23,52 \%)$ animais.

\section{Referências}

ANAI, L. A. Evolução da expressão de CD45+ e do leucograma de cães linfomatosos durante quimioterapia com o protocolo de Madison-Wisconsin. 2011. $51 \mathrm{f}$. Dissertação (Mestrado) - Faculdade de Ciências Agrárias e Veterinárias, Universidade Estadual Paulista, Jaboticabal, 2011.

AYRES, M. et al. Bioestat: Aplicações estatísticas nas áreas de ciências biológicas e médicas. Belém: Sociedade Civil Mamirauá, Brasília, 2008. 364 p.

BRANDÃO, C. V. S. et al. Tumor venéreo transmissível: estudo retrospectivo de 127 casos (1998-2000). Revista de Educação Continuada, CRMV-SP, São Paulo, v. 5, n.1, p. 25-31, 2002.

CAVALCANTI, R. L. et al. Aspectos epidemiológicos do TVT em cães atendidos no Hospital de Clínicas Veterinárias da Universidade Federal do Rio Grande do Sul. Acta Scientiae Veterinariae, Porto Alegre, v. 35, n.14, p.13431344, 2007.

CHUN, R. Lymphoma: which chemotherapy protocol and why? Topics in Companion Animal Medicine, New York, v. 24, n. 3 , p. $157-162,2009$.

CRUZ, G. D. et al. Metástase visceral de tumor venéreo transmissível em cão. Veterinária e Zootecnia, Botucatu, v. 16, n. 3, p. 465-470, 2009.

DAS, U.; DAS, A. K. Review of canine transmissible venereal sarcoma. Veterinary Research Communications, Pantnagar, v. 24, n. 8, p. 545-556, 2000.

FARO, A. M. et al. Avaliação hematológica em cães submetidos ao tratamento quimioterápico com sulfato de vincristina, prednisona e ciclofosfamida. Estudo experimental. Ars Veterinária, Jaboticabal, v. 24, n.1, p 001-008, 2008.

LANORE, D.; DELPRAT, C. Quimioterapia anticancerígena. São Paulo: Roca, 2004. p 53-170.

LIMA, E. R. et al. Frequência, aspectos clínicos, diagnóstico e tratamento de tumor venéreo transmissível (TVT) em cães atendidos no Hospital Veterinário da UFRPE. Medicina Veterinaria, Dois Irmãos, v. 5, n. 1, p. 24-29, 2011. animais. São Paulo: Roca, 2007. 300 p.

NAK, D. A clinic-pathological study on the effect of vincristine on transmissible venereal tumor in dogs. Journal of Veterinary Medicine A, Physiology, Pathology, Clinical Medicine, New Jersey, v. 52, p. 366370, 2005.

O'KEEFE, D. A.; HARRIS, C. L. Toxicology of oncologic drugs. Veterinary Clinics of North America: Small Animal Practice, Philadelphia, v. 20, n. 2, p. 483-504, 1990.

PAOLONI, M. C.; KHANNA, C. Comparative oncology today. Clinics of North America: Small Animal Practice, Philadelphia, v. 37, n. 6, p.1023-1032, 2007.

PEREZ, R. R. et al. A ação do decanoato de nandrolona (Deca-Durabolin ${ }^{\circledR}$ ) sobre parâmetros hematológicos e proteína total plasmática de ratos (Rattus rattus) com depressão medular induzida após administração de sulfato de vincristina (Oncovin $®)$. Ciência Rural, Santa Maria, v. 35 , n. 2, p. 589-595, 2005.

RODASKI, S.; DE NARDI, A. B. Quimioterapia antineoplásica em cães e gatos. São Paulo: Medvet Livros, 2007. p. 308

SILVA, M. C. V. et al. Avaliação epidemiológica, diagnóstica e terapêutica do tumor venéreo transmissível (TVT) na população canina atendida no hospital veterinário da UFERSA. Acta Veterinaria Brasilica, Mossoró, v.1, n.1, p. 28-32, 2007.

SIMERMANN, N. F. S. Sulfato de vincristina no tratamento do tumor venéreo transmissível frente à caracterização citomorfológica. 2009. 64 f. Dissertação (Mestrado em Ciências Animal) - Universidade Federal de Goiás, Goiânia, 2009.

SOBRAL, R. A.; TINUCCI, C. M.; CAMACHO, A. A. Occurrence of canine transmissible venereal tumor in dogs from the Jaboticabal region, Brazil. Ars Veterinária, Jaboticabal, v.14, p.1-10, 1998.

WITHROW, S. J.; MACEWEN, E. G. Withrow and macEwen's small animal clinical oncology. 4. ed. Philadelphia: Saunders, 2007. 163-188 p.

Recebido em: 04/07/2013 Aceito em: 06/06/2014

MORRIS, J.; DOBSON, J. Oncologia em pequenos 